\title{
3D PRINTING-PERIODONTAL PERSPECTIVE
}

\section{Periodontology \\ Dr. Deepti Rakesh Gattani}

Dr. Nupur Kar*

Professor , Department of Periodontology, Swargiya Dadasaheb Kalmegh Smruti Dental College and Hospital, Hingna Nagpur.

Post Graduate Student, Department of Periodontology, Swargiya Dadasaheb Kalmegh Smruti Dental College and Hospital, Hingna, Nagpur. *Corresponding Author

Post Graduate Student, Department of Periodontology, Swargiya Dadasaheb Kalmegh Smruti Dental College and Hospital, Hingna, Nagpur.

\section{ABSTRACT}

3 dimensional printing is a novel technique which helps in opening several new avenues of regenerative Periodontology. It aims to make several regenerative procedures customizable and feasible for the patients. This review mainly focuses on the periodontal applications of 3 dimensional printing and understanding how it might help us to better perform surgeries. To gain in depth knowledge of 3 Dimensional (3D) printing various techniques has also been explained .It is extremely essential to remain updated on the various technological advancements in the field of periodontal surgery so that we can provide the best possible care to our patients.

\section{KEYWORDS}

\section{Dimensional printing, Periodontics, Regenerative}

\section{INTRODUCTION}

Recent technological advances has changed the way dentists treat their patients in recent years, we have started using technological advances from nanotechnology to 3D printing to help us treat patients. 3D printing has recently made the headlines because of its possibility to regenerate organs this will treat the age old problem of organ transplant. $3 \mathrm{D}$ printing is the term used to describe additive manufacturing approach that builds material layer by layer ${ }^{1}$ as opposed to the subtractive manufacturing where 3 dimensional objects were made by successively cutting away from a block of material. "Digital workflow" in dentistry comprises of three elements; acquisition of data through scanning, processing of data using computer-aided design software (CAD), and use the information to build objects using computer-aided-manufacturing. In the manufacturing step, 3D printing(additive manufacturing) is becoming a fast alternative for substractive manufacturing. previously performed by subtractive manufacturing ${ }^{2}$.

3D printing is now being used in several fields of dentistry like Oral surgery for virtual planning in complex surgical cases or Prosthodontics for extra oral prosthesis but this review mainly focuses on its application in Periodontology.

\section{Techniques of $3 D$ printing}

Different techniques of $3 \mathrm{D}$ printing have been reported in literature with advantages and disadvantage of each technique. These include stereolithography, photopolymer jetting, selective laser sintering, fused deposition modeling, and powder binder printers.

Stereolithography is a rather new shaping technique that makes it possible the fabrication of complex 3D structures with a high dimensional accuracy. The newer part is built through layer by layer polymerization. ${ }^{4}$

In Photopolymer jetting technology, 3-dimensional objects are built by selective deposition of UV-curable composition only when required and composition is cured immediately by a UV radiation from a bulb source.

Selective Laser Sintering is a type of powder Bed fusion wherein Bed of powder polymer, resin or metal is targeted partially (sintering) or fully (melting) by a high power directional heating source such as laser that result to a solidified layer of fused powder. ${ }^{6}$

Fused deposition modeling is a low cost extrusion based 3D printing technology which fits very well in frame work of primary and secondary recycling. ${ }^{7}$ Here compositions are cured in their entirety immediately after deposition, by exposure to UV radiation from a bulb source.

\section{Advantages of $3 D$ printing}

1) Preoperative virtual planning and patient-specific $3 \mathrm{D}$ printing which seems to provide additional predictability in complex surgical cases ${ }^{2}$

2) To make customizable scaffold for patients with bony defects or for socket preservation. Customization can lead to perfect fit of scaffold within the bony defect, it can also help in measuring and calculating the amount of bone substitutes or xenograft or allograft to be used prior to surgery.

3) $3 \mathrm{D}$ printed multi-material components may be adopted as remedial solution for converting polymeric waste into useful functional parts with acceptable mechanical properties. ${ }^{7}$ This will lead to recycling plastic and generating less environmental pollution as $3 \mathrm{D}$ printing will create less waste.

The major limitation of $3 \mathrm{D}$ printing is the cost spent in generation of $3 \mathrm{D}$ objects. Use of $3 \mathrm{D}$ printing for individual implant design does not seem to be justified because standard planning procedures are usually sufficient.

\section{Applications of 3D printing in Periodontology}

Periodontal applications in 3D printing is usually less known, however they include applications in various forms like education models, scaffolds, socket preservation, sinus and bone augmentation, repairing periodontal bone defects and guided implant placement.

Although, 3D printing gives us precision and customization in periodontal surgery still, most of the periodontist hesitate to use it in routine practice due to the expensive nature of this procedure. However, due to the evolving nature of this technology the coming future will see it become more user-friendly and pocket friendly.

There are several periodontal uses of 3D printing, as it is a developing field many more are yet to follow however these are the major ones Education Models And Surgical Guide

Patients are usually apprehensive of surgeries and to explain procedures like implant, tissue and bone regenerative procedures, socket preservation educational models can be used.It can also be used by post graduation students for educational purposes.

Surgical guides are very important for performing a complex surgery, they help prepare the dentist better perform the surgery. Templates for alveolar ridge augmentation are fabricated using a combination of Cone-beam computed tomography (CBCT), CAD/CAM and 3D printing. Also customized templates are being widely used in the treatment of severe horizontal and vertical bony defects. ${ }^{8}$

\section{Socket Preservation}

The extraction or natural loss of tooth leads to loss of dimensions of alveolar ridge due to resorption. It has been reported in a systematic review that after tooth extraction, average reduction in alveolar bone 
width and height was $3.87 \mathrm{~mm}$ where maximum follow up was 7 months ${ }^{9}$.

Recent advancement in 3 dimensional printing has further eased the treatment protocol of socket preservation.

Several studies like Ghon BT et al. (2015), Kijartorn et al. (2017) have inserted a 3D polycaprolactone (PCL) scaffold in fresh extraction sockets which allowed normal bone healing, as compared to extraction sockets without the scaffold. ${ }^{10}$

\section{Three-dimensional Printed Scaffold For Bone And Tissue Regeneration}

In the treatment for periodontal bone defect, Rasperini et al first reported use of 3D-printed bioresorbable polymer scaffold for periodontal tissue regeneration. ${ }^{11}$ Advancements in three-dimensional (3D) imaging acquisition technologies such as CBCT and scaffold fabrication methods such as $3 \mathrm{D}$ bioprinting have resulted in personalized scaffolding constructions based on individual patient's data. However, 'fiber-guiding' scaffold designs utilize the topographical cues present to guide ligamentous fibers to form towards the root surface to improve tooth support ${ }^{8}$.

Scaffold material used is PCL hydroxapatitie which is a resorbable material. However, recently the micropatterned fiber-guiding scaffolds were tested in an alveolar fenestration animal Model ${ }^{17}$. The results have demonstrated strong potential of the combined strategies of fiber-guiding scaffolds and gene therapy in restoring the lost periodontium. ${ }^{8}$

\section{Tissue Regeneration}

In contrast to conventional single-system methods of fibrous tissue formation, by using $3 \mathrm{D}$ printing, we can use biomaterial and biologics for tissue regeneration instead of tissue replacement. ${ }^{11}$ Dentistry is now moving from tissue replacement to tissue regeneration.

\section{Bone Regeneration}

Personalized scaffolding is being increasingly used more in the current times for procedures such as ridge reconstruction for site development where implant can be later placed. 3D-printed diagnostic models and preoperative templates are widely used for ridge augmentation where severe vertical and horizontal bony defects are present. ${ }^{8}$

In case reports mentioned Lihong Lie et al (2019) used a 3D printer and CBCT datasets to produce a photosensitive resin bony anatomy replica. The patient's blood was centrifuged to obtain advanced platelet-rich fibrin (A-PRF) and injected platelet-rich fibrin (I-PRF), then mixed with Bio-Oss and packed onto the $3 \mathrm{D}$ replica to form the ideal shape. The replica was placed at the pre-planned site without any changes. The A-PRF membrane was placed over the replica as well as a collagen membrane.

This implicates that 3D printing along with PRF can be used to preplan surgeries and avoid wastage of material. Since, the 3D replica can be directly placed in the patient's mouth, the time require fir the surgery is notably reduced. Additionally, the surgeons could surgically rehearse the surgery with the personalized scaffold.

\section{Sinus Augmentation}

Carlo Mangano et al. (2015) did a study where a custom-made 3D synthetic bone substitute was evaluated when used as scaffold for sinus augmentation procedures in an animal model ${ }^{14}$. In vivo human studies are lacking so the present data is insufficient to be generalized for applications in this field.

\section{Bone Augmentation}

Tomoki Sumada et al. (2015) did a study where three-dimensional printing was used to make Titanium devices as membranes for bone augmentation in implant placement. These custom-made devices were constructed by a selective laser melting method with titanium. The custom-made devices not only fit better than the conventional counterpart they required less operating time. ${ }^{16}$

\section{Three-dimensional Printing For Guided Implant Placement Root-analogue Implants (RAIs)}

Immediate implant placement has an advantage of reduced treatment time, immediate tooth replacement, preservation of the normal periodontal and gingival structures. It is thus a viable treatment option in the aesthetic region. However, the pre fabricated implants available have a different shape than that of the socket. 3D printing can be used to custom design implant (root analogue implant) providing an acceptable socket fit, leading to better osseointegration. Cone-beam computed tomography can be used to scan and manufacture the customizable prefabricated implant.

Francesco Guido Mangalo (2014) did a study where CBCT acquisition and 3D image conversion, combined with the direct laser metal sintering (DLMS) implants process, allow the fabrication of custommade, RAIs. At the 1-year follow-up, no implants were lost, for a survival rate of $100 \%$. All implants were stable with no signs of infection. ${ }^{16}$

Bose MWH et al (2020) did a retrospective study where they evaluated 107 RAIs. Survival of implants was verified at follow-up examinations. The study reported that 6 RAIs failed, of which 4 did not show osseointegration in the healing period and 2 were lost after prosthetic delivery. The calculated survival rate for RAIs which is $94.4 \%$ when compared to the survival rate of $94.6 \%$ considering 10 year follow-up data of screw-shaped implants

Since the RAI has been evaluated retrospectively need for prospective clinical trials in this field is recommended.

\section{CONCLUSION}

With more advances in bioprinting has allowed delivery of cells, extracellular matrices, genes and/or biological agents onto polymeric, ceramic , and natural biomaterials. ${ }^{8}$ These developments in the cellular level will help us make regenerative therapies predictable and reliable. It will shift the paradigm of dentistry from restorative and replacement to regenerative. Several newer techniques are upcoming with better precision and prognosis than conventional techniques.

\section{REFERENCES}

1. Barazanchi, A., Li, K. C., Al-Amleh, B., Lyons, K, \& Waddell, J N (2017) Additive technology: update on current materials and applications in dentistry. Journal of

2. Katkar, R. A., Taft, R. M., \& Grant, G. T. (2018). 3D volume rendering and 3D printing (additive manufacturing). Dental Clinics, 62(3), 393-402

3. Gul, M., Arif, A., \& Ghafoor, R. (2019). Role of three-dimensional printing in periodontal regeneration and repair: literature review. Journal of Indian Society of Periodontology, 23(6), 504.

4. Chartier, T., Badev, A., Abouliatim, Y, Lebaudy, P., \& Lecamp, L. (2012) Stereolithography process: influence of the rheology of silica suspensions and of the medium on polymerization kinetics-cured depth and width. Journal of the European Ceramic Society, 32(8), 1625-1634

5. Napadensky, E. (2005). Ink-Jet 3D Printing of Photopolymers Materials: An Emerging Rapid Prototyping Technology. In RadTech Europe 2005 Conference \& Exhibition, Barcelona, Spain

6. Dizon, J. R. C., Espera Jr, A. H., Chen, Q., \& Advincula, R. C. (2018).Mecanical characterization of 3D-printed polymers. Additive Manufacturing, 20,44-67. Singh R, Kumar R, Singh P. Prospect of 3D Printing for Recycling of Plastic Product to Minimize Environmental Pollution

8. Yu, N., Nguyen, T., Cho, Y. D., Kavanagh, N. M., Ghassib, I., \& Giannobile, W. V. (2019). Personalized scaffolding technologies for alveolar bone regenerative medicine. Orthodontics \& craniofacial research, 22, 69-75.

9. Van der Weijden, F., Dell'Acqua, F., \& Slot, D. E. (2009). Alveolar bone dimensional changes of post extraction sockets in humans: a systematic review. Journal of clinical periodontology, 36(12), 1048-1058

10. Goh, B. T., Teh, L. Y., Tan, D. B. P., Zhang, Z., \& Teoh, S. H. (2015). Novel 3 D polycaprolactone scaffold for ridge preservation-a pilot randomised controlled clinical trial. Clinical oral implants research, 26(3), 271-277

11. Park, C. H., Rios, H. F., Taut, A. D., Padial-Molina, M., Flanagan, C. L., Pilipchuk, S. P., ... \& Giannobile, W. V. (2014). Image-based, fiber guiding scaffolds: a platform for ... \& Giating tin

2.

, J, Giannobile WV. Micropatterned scaffolds with immobilized growth factor genes regenerate bone and periodontal ligament like tissues. Advanced healthcare materials 2018 Nov; $7(22): 1800750$

13. Lei, L., Yu, Y., Ke, T., Sun, W., \& Chen, L. (2019). The application of three-dimensional printing model and platelet-rich fibrin technology in guided tissue regeneration surgery for severe bone defects. Journal of Oral Implantology, 45(1), 35-43.

14. Mangano, C., Barboni, B., Valbonetti, L., Berardinelli, P., Martelli, A., Muttini, A.,... \& Mattioli, M. (2015). In vivo behavior of a custom-made 3D synthetic bone substitute in sinus augmentation procedures in sheep. Journal of Oral Implantology, 4I(3), 240-250.

15. Sumida, T., Otawa, N., Kamata, Y. U., Kamakura, S., Mtsushita, T., Kitagaki, H., ... \& Yamaguchi, A. (2015). Custom-made titanium devices as membranes for bone augmentation in implant treatment: clinical application and the comparison with conventional titanium mesh. Journal of Cranio-Maxillofacial Surgery, 43(10), 2183-2188.

6. Mangano, F. G., De Franco, M., Caprioglio, A., Macchi, A., Piattelli, A., \& Mangano, C. (2014). Immediate, non-submerged, root-analogue direct laser metal sintering (DLMS) implants: a 1-year prospective study on 15 patients. Lasers in Medical Science, 29(4), 1321-1328.

17. Böse, M. W. H., Hildebrand, D., Beuer, F., Wesemann, C., Schwerdtner, P., Pieralli, S \& Spies, B. C. (2020). Clinical Outcomes of Root-Analogue Implants Restored with Single Crowns or Fixed Dental Prostheses: A Retrospective Case Series. Journal of Clinical Medicine, 9(8), 2346. 